\title{
Research
}

\section{Interventions to reduce primary care delay in cancer referral:}

\author{
a systematic review
}

\begin{abstract}
\section{Background}

Reducing delay in the primary care part of the cancer care pathway is likely to improve cancer survival. Identifying effective interventions in primary care would allow action by primary healthcare professionals and local commissioners to reduce delay.
\end{abstract}

\section{Aim}

To identify interventions that reduce primary care delay in the referral of patients with cancer to secondary care.

\section{Design and setting}

Systematic review in primary care.

\section{Method}

Eight electronic databases were searched using terms for primary care, cancer, and delay. Exclusion criteria included screening and the 2 week-wait referral system. Reference lists of relevant papers were hand searched. The quality of each paper was assessed using predefined criteria, and checked by a second reviewer.

\section{Results}

Searches identified 1798 references, of which 22 papers were found to meet the criteria. Interventions concerning education, audit and feedback, decision support software and guideline use, diagnostic tools, and other specific skills training were identified. Most studies reported a positive effect on their specified outcomes, although no study measured a direct effect on reducing delay.

\section{Conclusion}

There was no evidence that any intervention directly reduced primary care delay in the diagnosis of cancer. Limited evidence suggests that complex interventions, including audit and feedback and specific skills training, have the potential to do so.

\section{Keywords}

early diagnosis; intervention studies: neoplasms; primary health care.

\section{INTRODUCTION}

Total delay in cancer diagnosis (from the first symptom noticed by the patient to a diagnosis or treatment) by more than 3 months has been found to have an adverse effect on survival in breast cancer. Evidence for other cancers, and which component of the patient pathway is most significant in impairing survival, is lacking. ${ }^{2}$ UK government policy to reduce diagnostic delay in all cancers has focused on public education, screening, and referral delay, ${ }^{3}$ the latter consisting of doctor delay (first consultation to referral by a doctor) and hospital delay (referral by a doctor to diagnosis). ${ }^{4}$ In 1999, the government introduced an initiative for breast cancer extended to all cancers in 2000, known as the 2-week-wait (2ww) rule. ${ }^{5}$ This states that if a GP suspects a diagnosis of cancer then they are required to send a referral within 24 hours and the secondary care provider to give an appointment for consultation within 2 weeks. In 2005, the National Institute for Health and Clinical Excellence (NICE) introduced referral guidelines for primary care concerning symptoms that are suspicious of cancer These initiatives were prompted by studies suggesting that cancer survival in the UK was poor compared to other European countries, ${ }^{6}$ although the inference that care is 'substandard' continues to be questioned. ? In this review, the term 'delay is used in a non-pejorative manner to represent a potential reduction in the time interval from presentation with a symptom or sign in primary care to referral to secondary care.

A review of audits of the governmentinitiated $2 \mathrm{ww}$ found that many audits were not well reported and their results appeared to show that the guidelines are not uniformly complied with. ${ }^{8}$ No conclusions were made concerning the effectiveness of the $2 \mathrm{ww}$ intervention in reducing diagnostic delay across the whole pathway. While arguments continue concerning the effectiveness of the intervention, NHS commissioners are obliged to follow government policy and take account of NICE guidance in their commissioning decisions.

Austoker et al conducted a systematic review of interventions to reduce patient delay (time from noticing a symptom to first doctor consultation), ${ }^{4}$ which covered both individual and community-level interventions. The authors found no studies that stated reducing delay as an explicit outcome, but found limited evidence for interventions to raise individual and community awareness and encourage early presentation, proxy measures for reduced delay.

Studies concerning medical care in general, ${ }^{10}$ and breast cancer specifically, ${ }^{11}$ found that good clinical outcomes may be achieved through improving knowledge, leading to an improvement in attitudes and change in behaviour. This implies that interventions to improve knowledge of particular symptoms or diagnostic skills of primary healthcare professionals (PHPs) may be used as proxy measures for reduced
G Mansell, MSc, research assistant; M Shapley, DM, FRCGP, GP and senior research fellow: JL Jordan, MSc, MA, research information manager; KP Jordan, PhD, reader in biostatistics, Primary Care Sciences, Keele University, Keele. Address for correspondence

Miss Gemma Mansell, Arthritis Research UK Primary Care Centre, Primary Care Sciences, Keele University, Keele, Staffordshire ST5 5BG.
E-mail: g.mansellacphc.keele.ac.uk

Submitted: 4 January 2011; Editor's response: 23 February 2011; final acceptance: 27 April 2011. (c)British Journal of General Practice

This is the full-length article (published online 28 Nov 2011) of an abridged version published in print. Cite this article as: Br J Gen Pract 2011. DOI: 10.3399/bjgp11X613160 


\section{How this fits in}

Primary care is often the first point of call for patients with symptoms that they think may be cancer related. Reducing delay in this section of the cancer care pathway may help to improve cancer survival. This review shows that there is only limited evidence for interventions in primary care.

primary care delay. In this review, primary care delay is defined as the time interval from the patient first presenting to a PHP to referral to secondary care.

Primary care has a very important role in the UK, as it is often the first point of call for a patient. Many parts of the consultation have the potential for interventions that would reduce delay in the cancer pathway. The aim of this review is to identify interventions that reduce primary care delay and have the potential to be commissioned locally or enacted by PHPs.

\section{METHOD}

\section{Search strategy}

Eight electronic databases were searched from inception to March 2010:

- MEDLINE (from 1950)

- Embase (from 1980)

- Cumulative Index to Nursing and Allied Health Literature (CINAHL) (from 1981)

- PsycINFO (from 1806)

- Health Management Information Consortium (HMIC) (from 1919)

- Web of Science (from 1970)

- Education Resources Information Centre (ERIC) (from 1966)

- Cochrane Library (from 1993).

The search terms included thesaurus and free-text terms for primary care, cancer, and delay. Specific types of intervention such as education and training were also included, as these were likely to give proxy measures for delay. An example of the search used in MEDLINE is given in Box 1. Once relevant articles from these databases had been identified, their reference lists were hand searched, and citation checking was performed to find any more papers that met the inclusion criteria. The reference lists of any relevant reviews were also checked for extra papers. Experts in the field were contacted for additional articles. All references were either downloaded or entered by hand into reference-management software.

\section{Eligibility criteria}

Any primary study that examined an intervention aimed at reducing primary care delay in the diagnosis of cancer was included. The study needed to have been carried out in a primary care setting, with an outcome of reducing delay, and had to include fully trained PHPs as participants. All study types were included. No limits were placed on date ranges or type of cancer.

\section{Exclusion criteria}

The following exclusion criteria were applied:

- studies that looked to improve screening or self-examination rates, as these involve asymptomatic patients and the review aimed to examine studies of presented symptoms

- studies not carried out in a primary care setting;

- studies of healthcare professionals other than fully trained general medical practitioners and practice nurses;

- studies with populations in which the effect on primary care professionals was not analysed separately, as it was not possible to distinguish the PHPs from other participants such as medical students and secondary care professionals. Similarly, studies involving community, primary, and secondary care, in which it was not possible to separate the primary care component, were also excluded;

- studies on the $2 \mathrm{ww}$, as this service cannot currently be decommissioned by local PHPs and commissioners; and

- non-English-language papers, due to resource constraints.

\section{Study selection and assessment of bias}

One reviewer screened firstly the titles of all downloaded references, and then the abstracts, in order to remove any papers that did not meet the inclusion criteria. The same reviewer then obtained full texts of all remaining papers. A second reviewer performed checks on $25 \%$ of the abstracts and all of the full-text articles, to ensure that the appropriate papers were being included. A $97 \%$ concordance rate was found for the abstract check, and an 88\% concordance rate was achieved for the full-text articles.

The quality of the articles was assessed independently by both reviewers, using a 


\section{Box 1. Search strategy (example taken from the MEDLINE database)}

“exp*. FAMILY PRACTICE OR exp. PRIMARY HEALTH CARE OR exp. PHYSICIANS, FAMILY OR exp. COMMUNITY HEALTH SERVICES OR "general practi*"+ OR GP OR (primary ADJ2 $\ddagger$ care) OR (family ADJ2 physician*) OR (family ADJ2 practi*) OR (family ADJ2 doctor*) OR (community ADJ2 (service* OR care)) OR (general ADJ2 physician*)"

AND

“exp. NEOPLASMS OR neoplasm* OR cancer* OR carcinoma* OR malignan* OR lesion* OR tumo?§r

AND

“educat* OR exp. EDUCATION, MEDICAL, CONTINUING OR skill* OR train* OR teach*

AND

(earl* ADJ2 diagnos*) OR (earl ADJ2 detect*) OR (delay* ADJ2 diagnos*) OR (delay* ADJ2 detect*) OR (suspicious OR suspect*) OR (earl ADJ2 recog*) OR (delay ADJ2 recog*) OR (present* ADJ2 symptom*) OR (delay* ADJ2 consult*)"

*exp. = exploded thesaurus term - picks up all articles that contain the particular terms as a keyword. t truncation - picks up various different word endings. $f$ adj = adjacent - picks up words next to each other in a document ladj3 = picks up words that have up to 3 words in between them). $\S ?=$ picks up alternative spellings.
Australia, four in the US, one in Canada, one in Egypt and Tunisia, and one in Pakistan. Studies were heterogeneous in terms of methodology and outcome, with no studies directly examining the primary outcome of reducing delay in the cancer care pathway. Instead, the outcomes included improved knowledge, improved observational skills or diagnostic accuracy, and improved referral rates. The types of intervention included were education ( $n=149$ ), audit and feedback ( $n=143$ ), decision-support software and guideline use ( $n=145$ ), diagnostic tools ( $n=$ 144), and other specific skills training ( $n=141)$

Overall, only six studies (all RCTs) scored above the study thresholds for good quality. Common problems across the articles included: selection bias, less than 1 year's follow-up of participants, small sample size, high dropout rate, and a lack of detail as to how the intervention was administered.

\section{Intervention type}

Education. Four before-and-after studies used traditional lecture settings, $14,15,29,30$ and one used a training session ${ }^{16}$ to try to improve knowledge of risk factors and diagnostic ability among participants. None of these studies were rated as good quality. All five involved similar assessments (preand immediate post-test), and all showed some improvement in knowledge of risk factors and diagnostic ability. The RCTs included used a variety of educational interventions including lectures, videos, and an internet triage package. ${ }^{18,19,21,22}$ The interventions were compared to control groups that received no intervention. They were of mixed quality and provided inconsistent evidence. The two studies that reported a positive outcome of improved diagnosis and management did not see the improvement continue to the end of the study period. ${ }^{21,22}$ Overall, studies rated as high quality reported poorer outcomes than those rated as low quality, and positive outcomes were only seen in the short term.

Audit and feedback. Three studies attempted to use audit and feedback to try to decrease primary care delay. One highquality RCT, ${ }^{28}$ where participants either did or did not receive feedback, reported improvement in the recording of clinical information but not in diagnostic accuracy. The low-quality before-and-after studies did show significant improvement in outcomes. ${ }^{17,33}$

\section{Decision-support software and guideline} use

Four studies, including three good-quality 


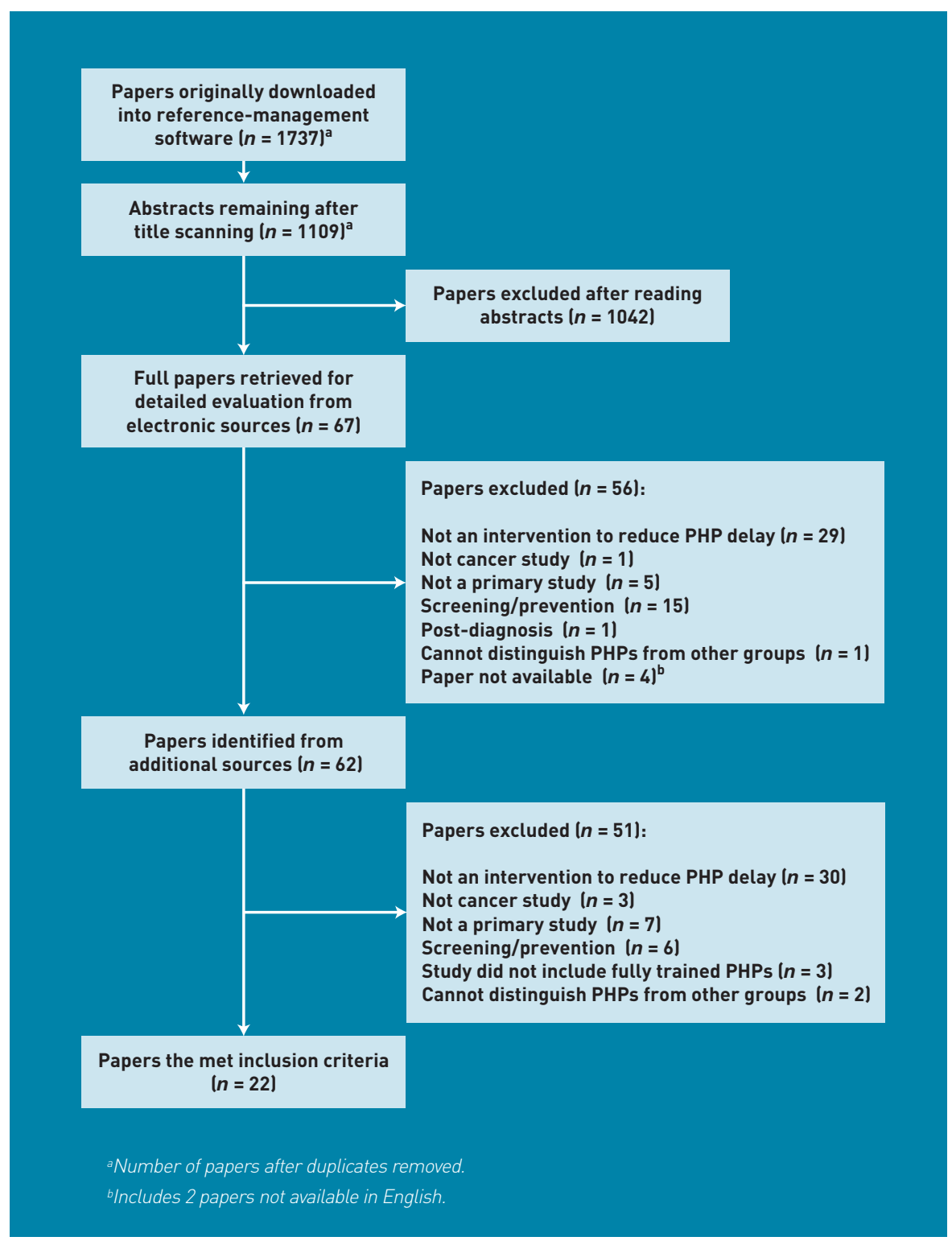

Figure 1. Flowchart of search.
RCTs, tested computer-based interventions to change PHP behaviour. 20,23,26,32 These studies were designed to improve the quality of referrals to secondary care as a proxy to reducing referral delay. Jiwa et al investigated the effectiveness of an educational outreach visit, ${ }^{23}$ an electronic referral pro forma', or both, on changing the type of colorectal referrals to secondary care. The control group did not receive an intervention. Neither intervention component was found to improve the appropriateness of referrals to secondary care, although the pro forma resulted in better documentation of those patients who were referred. Logan et al developed a prompt to improve the completeness of investigation and treatment of irondeficiency anaemia (IDA). ${ }^{26}$ The control group received a prompt to investigate the possible cause of the patient's IDA but no further information. This intervention was not shown to be effective in changing referral behaviour, but prescriptions of iron therapy increased in the intervention group. Wolters et al conducted a distance learning programme for prostate-specific antigen (PSA) testing and lower urinary tract symptoms. ${ }^{32}$ The authors found a decrease in urology referrals and a non-statistically significant rise in PSA testing, contrary to the outcome expected from the decision support tree.

In a low-quality before-and-after study, Gerbert et al devised software to improve triage of skin lesions, which included a decision tree, clinical information form, and support features. ${ }^{20}$ The authors found that participants made fewer incorrect decisions on clinical pictures when using the software.

A low-quality before-and-after study by Nekhlyudov et al tested an intervention to improve breast cancer outcomes by improving the use of breast-symptomrelated guidelines. ${ }^{11}$ Although the study was found to increase guideline use, no effect on knowledge or attitudes was found.

Diagnostic and assessment tools. Four studies tried to improve particular skills or the use of equipment to increase diagnostic accuracy, but none were deemed to be good quality.

Moreno-Ramirez et al's before-and-after study of skin cancer used teledermatology to investigate whether clinical examination or dermoscopy was more effective in improving the appropriateness of referrals to secondary care. ${ }^{27}$ Dermoscopic teleconsultations were found to lead to more relevant referrals. Khan conducted a feasibility study to explore whether an assessment tool using the CAPER score la clinical prediction rule) for colorectal cancer would be useful in general practice. ${ }^{24}$ It was found that uptake of the tool was low.

Argenziano et al's RCT'13 involved PHPs first attending a 1-day training course describing the ABCD (Asymmetric, Border, Colour, Diameter) rule for skin cancer ${ }^{34}$ and a three-point checklist. They were then randomly assigned to assess patients with skin lesions, either by standard clinical examination or by dermoscopy, and these assessments were checked for accuracy, by dermatologists. Dermoscopy improved triage, referral sensitivity, and accuracy, and led to participants being less likely to miss skin cancers in comparison to standard clinical examination. Westerhoff et al's RCT evaluated an educational intervention for improving melanoma diagnosis using 


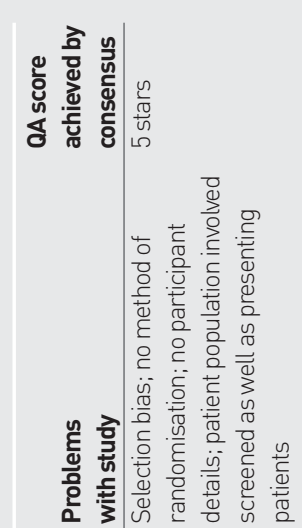

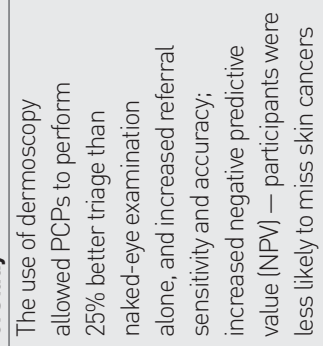

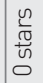

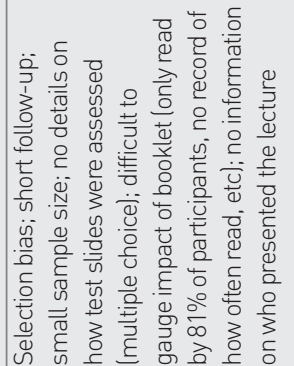

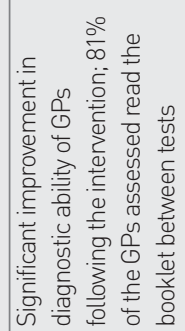

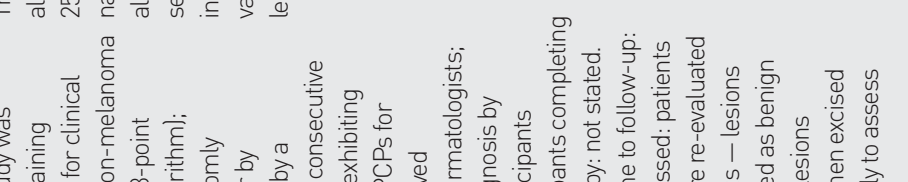

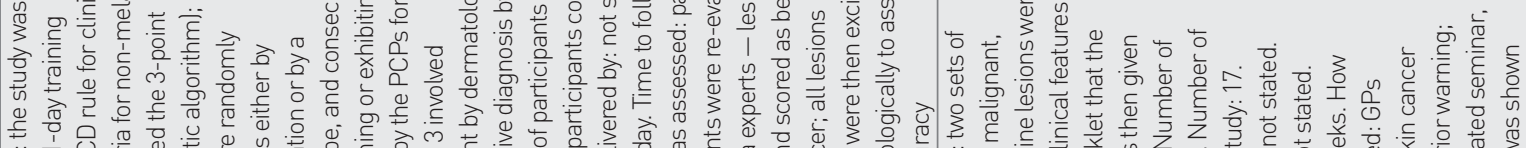

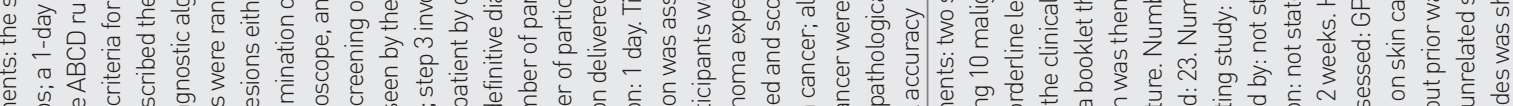

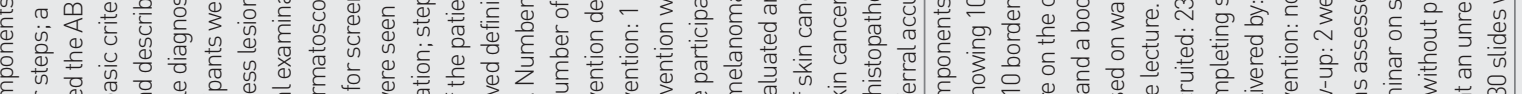

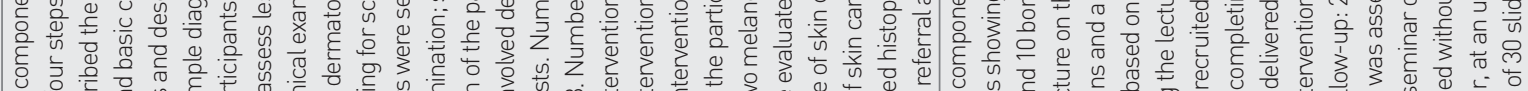

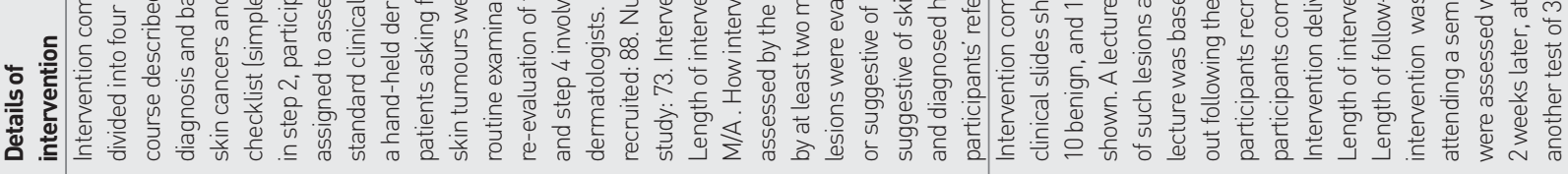

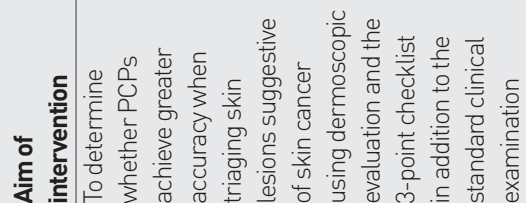

彭离

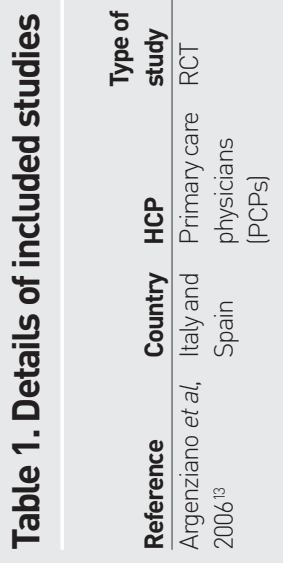

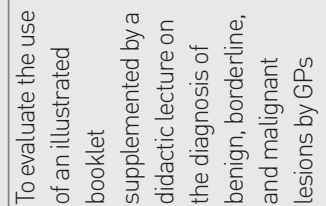

$\frac{\cdot \frac{5}{5}}{\text { ज }}$

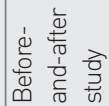

웅

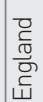

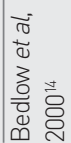




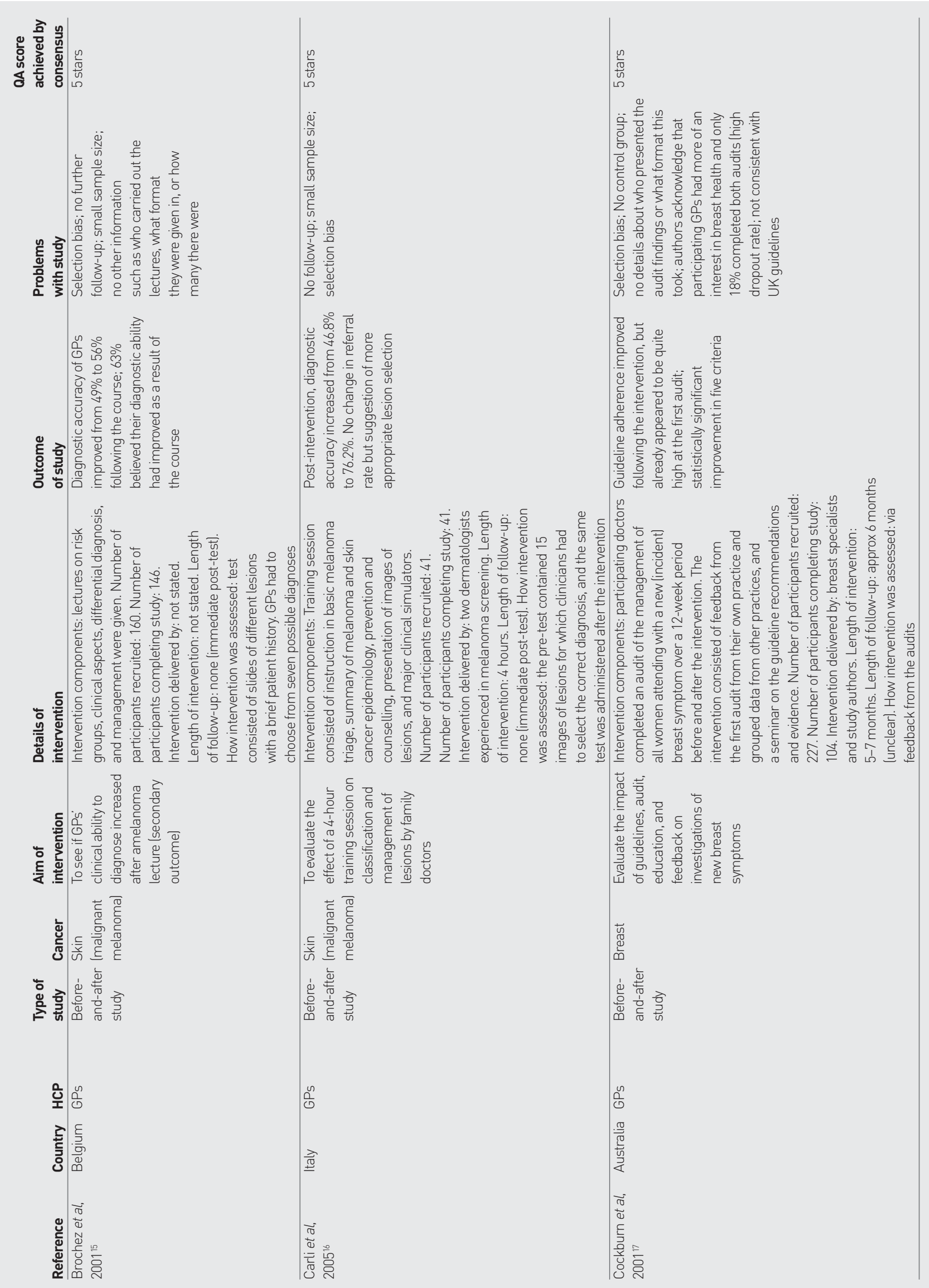




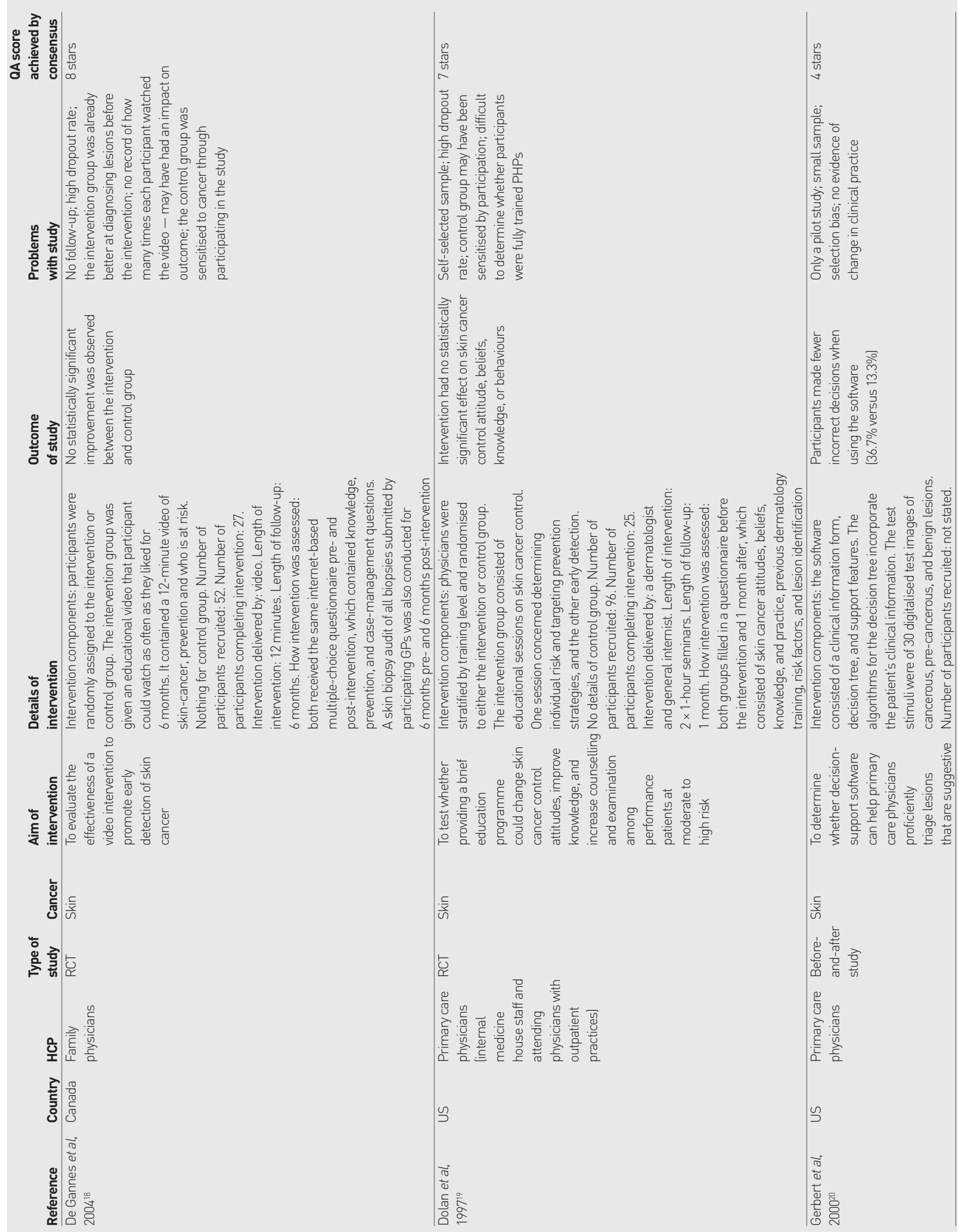




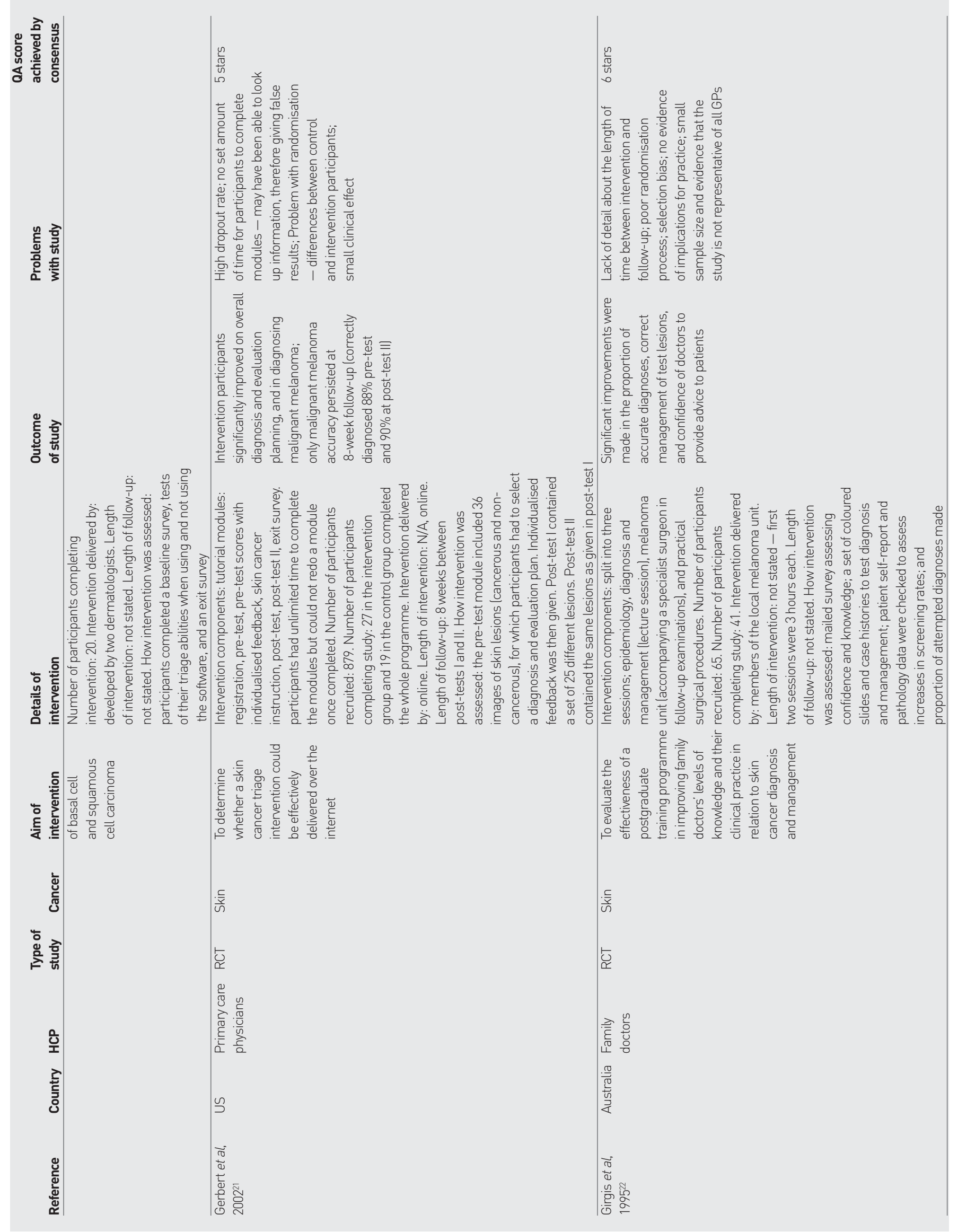


III

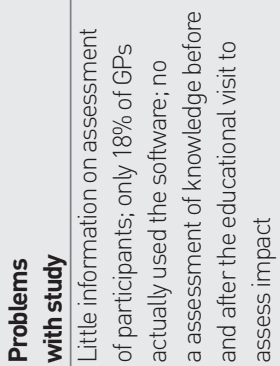

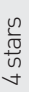
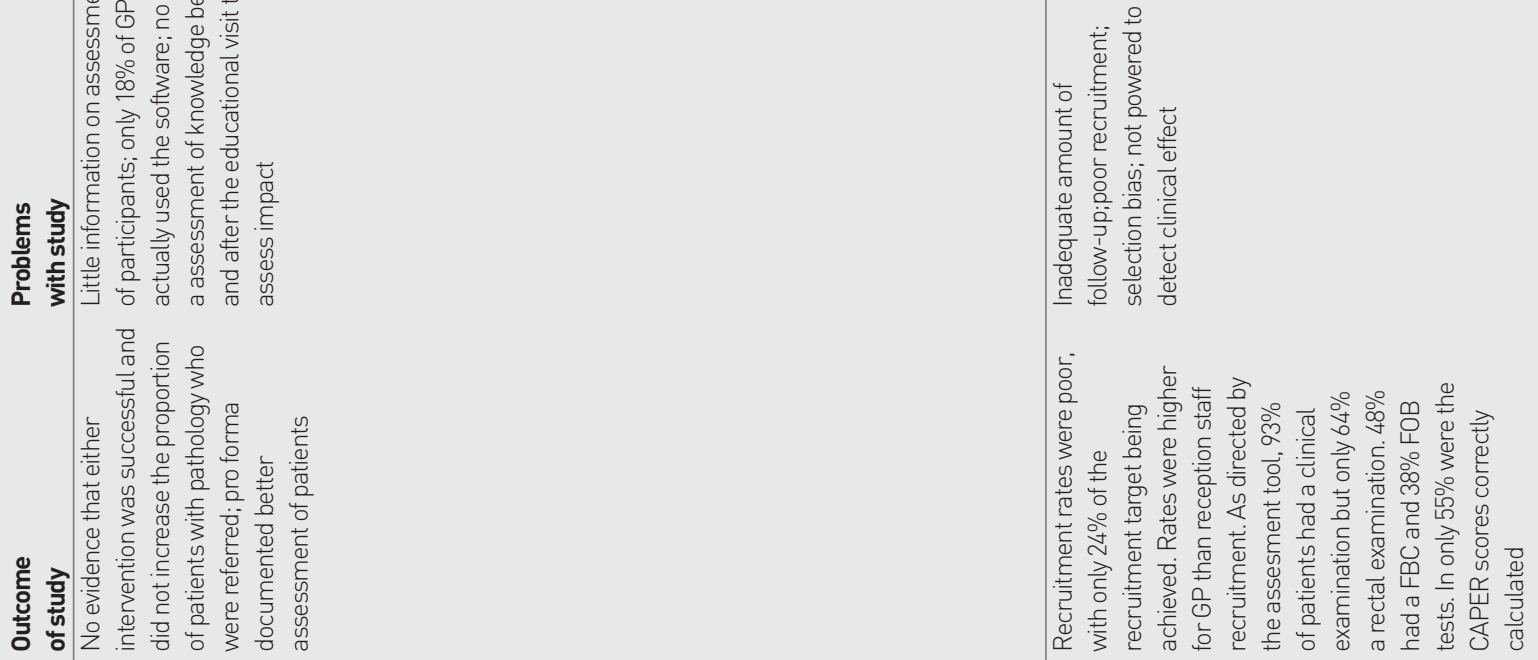

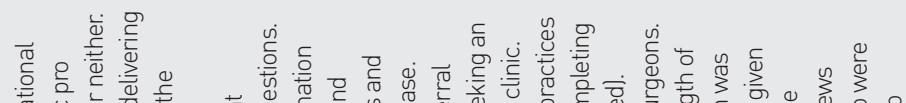

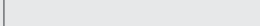
$\begin{array}{llll} & \\ 0\end{array}$

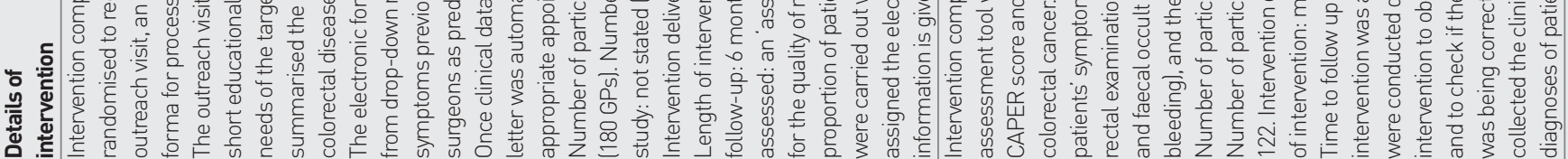
ใดำ
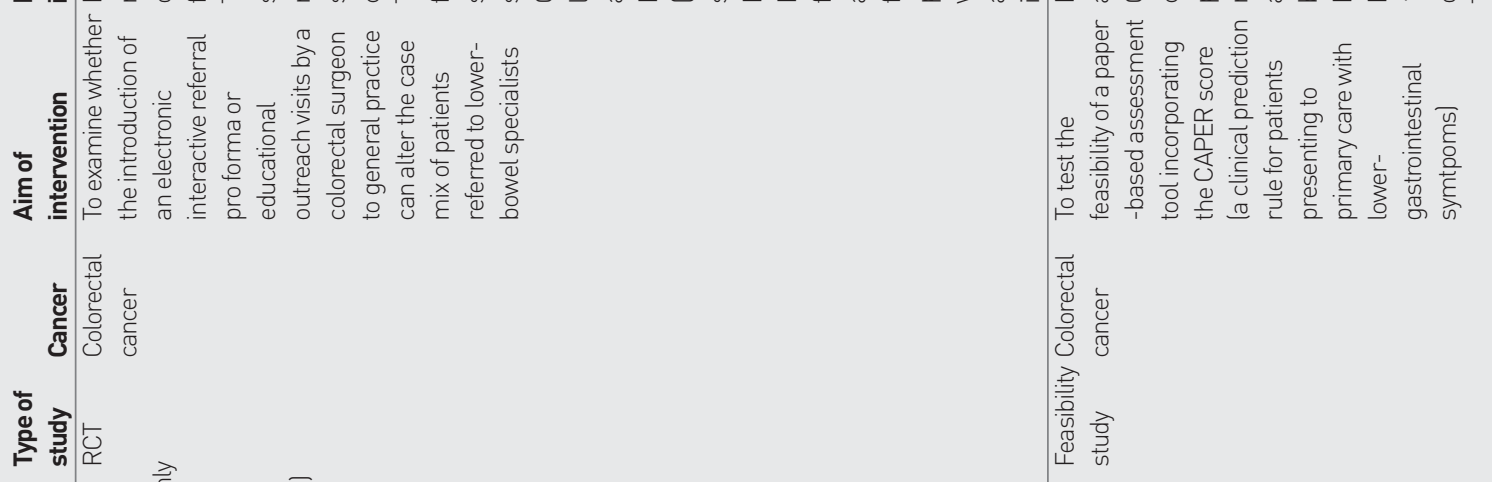

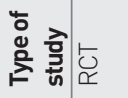

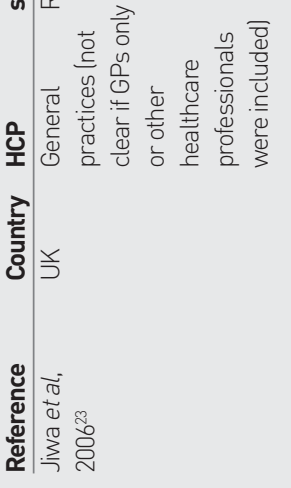




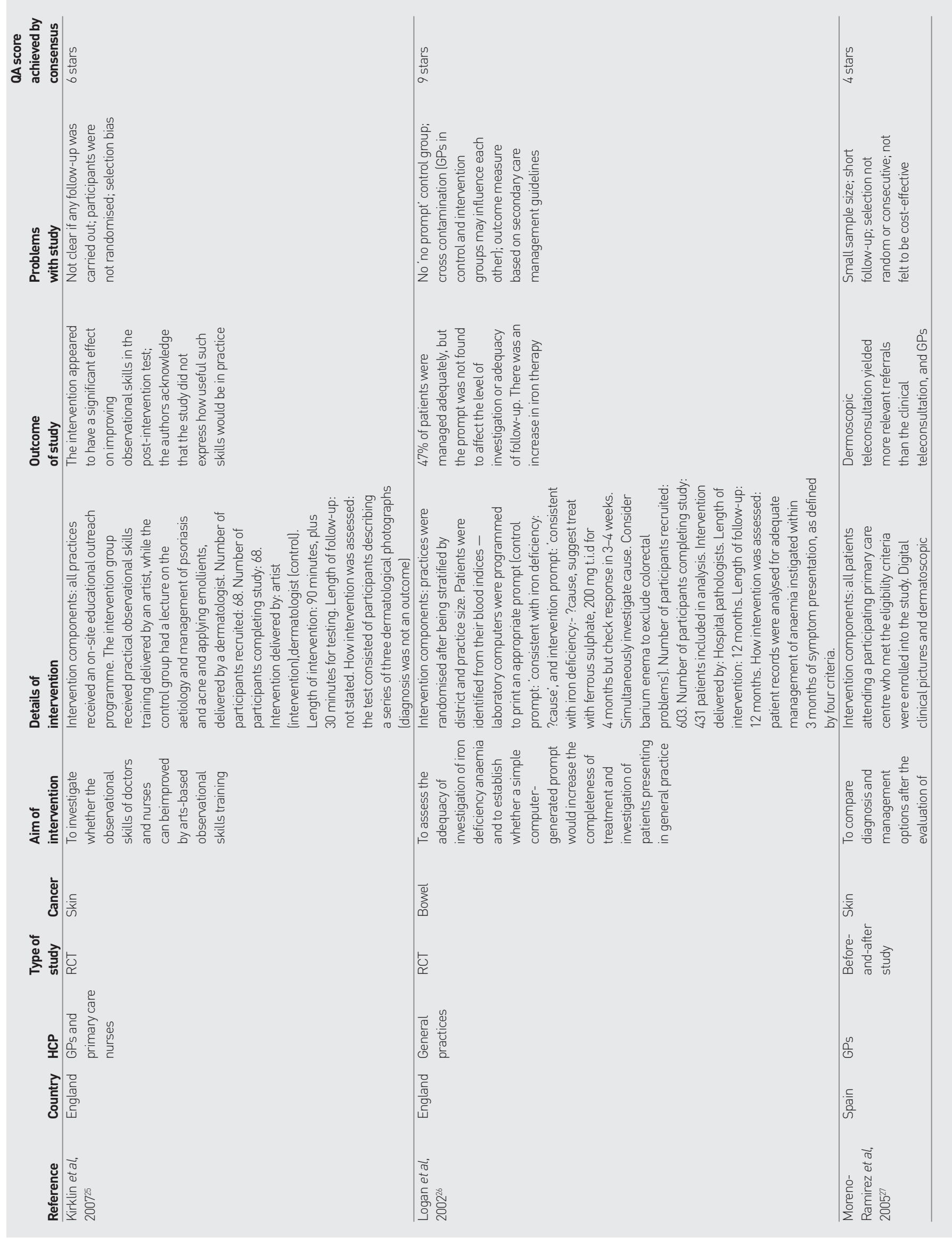




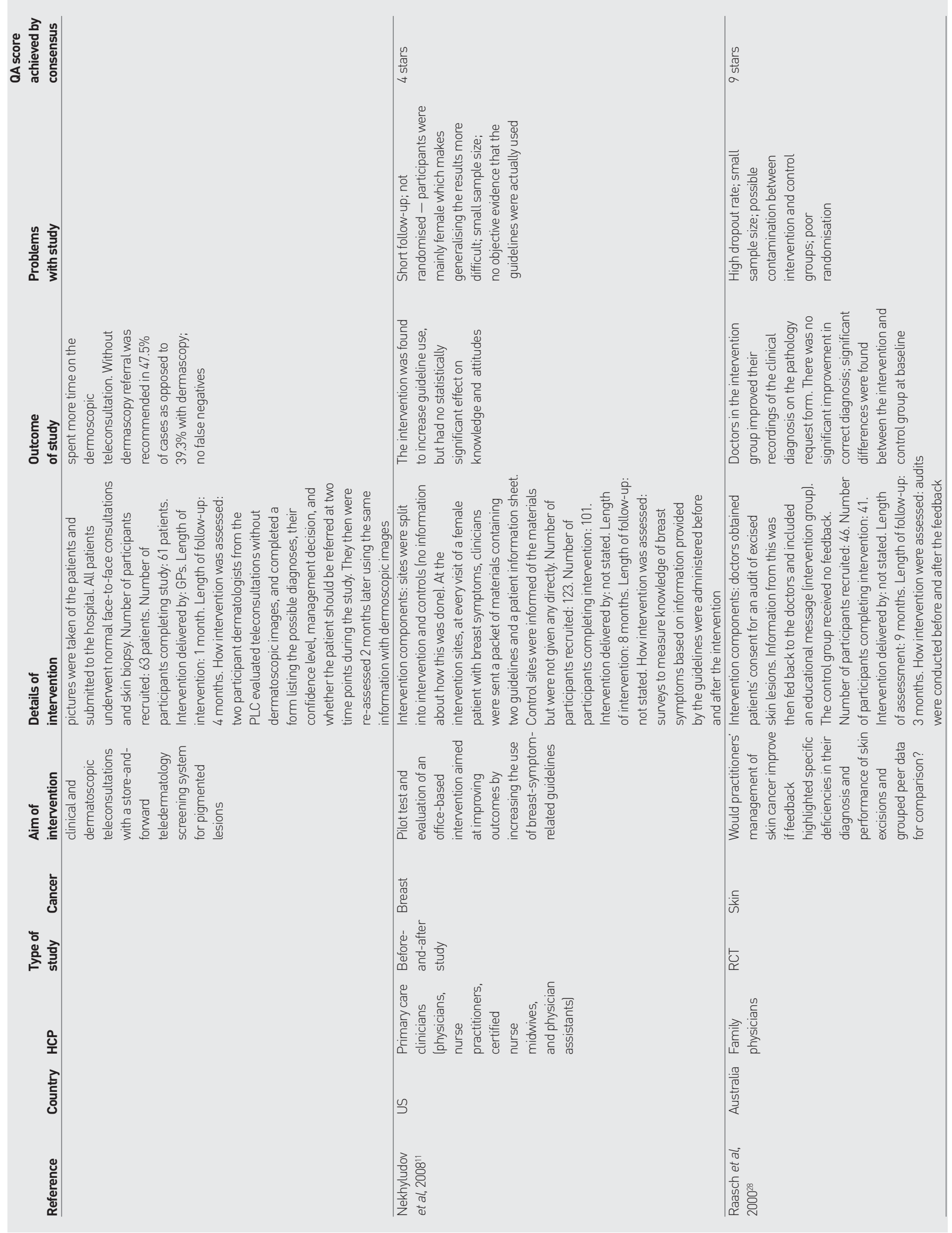




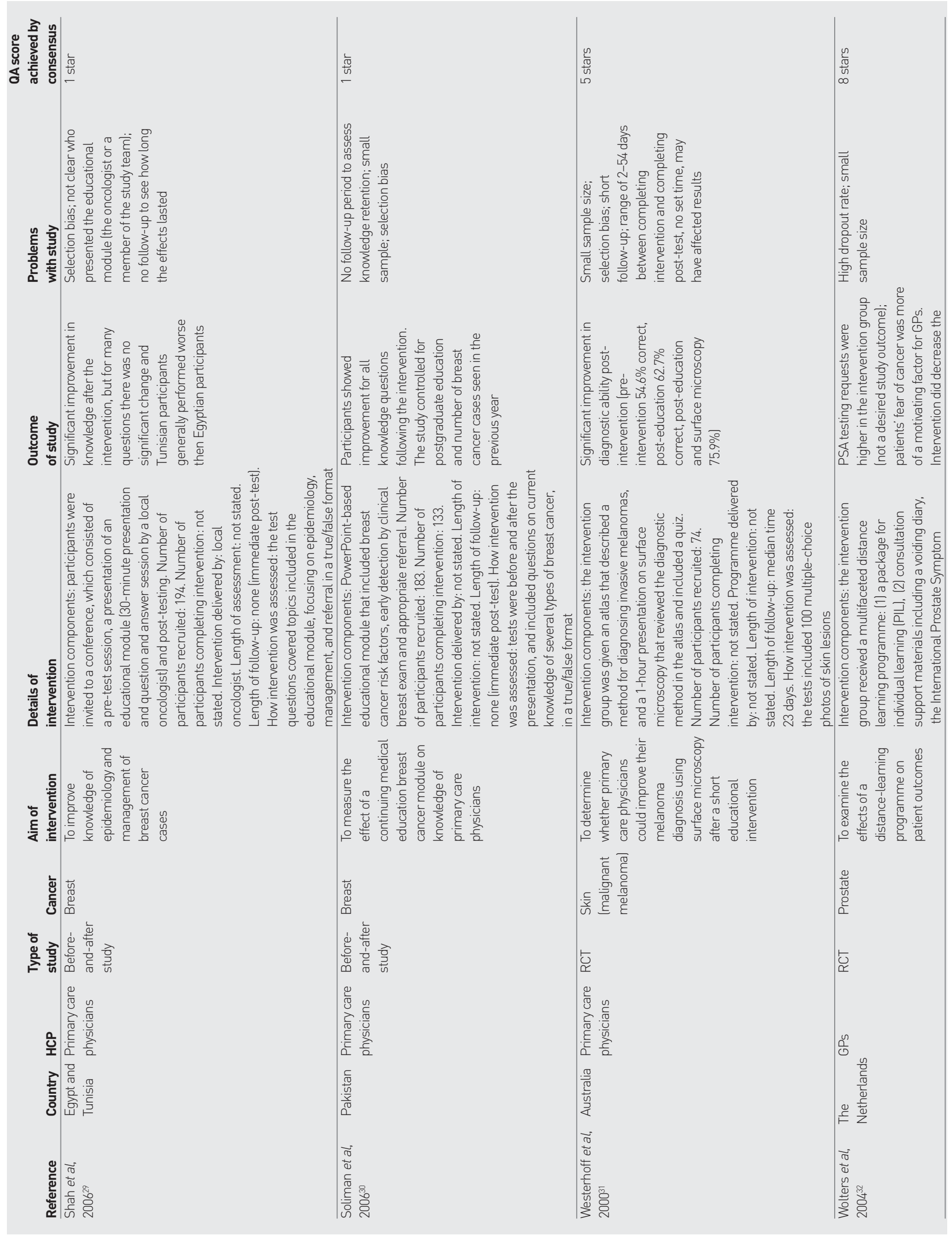




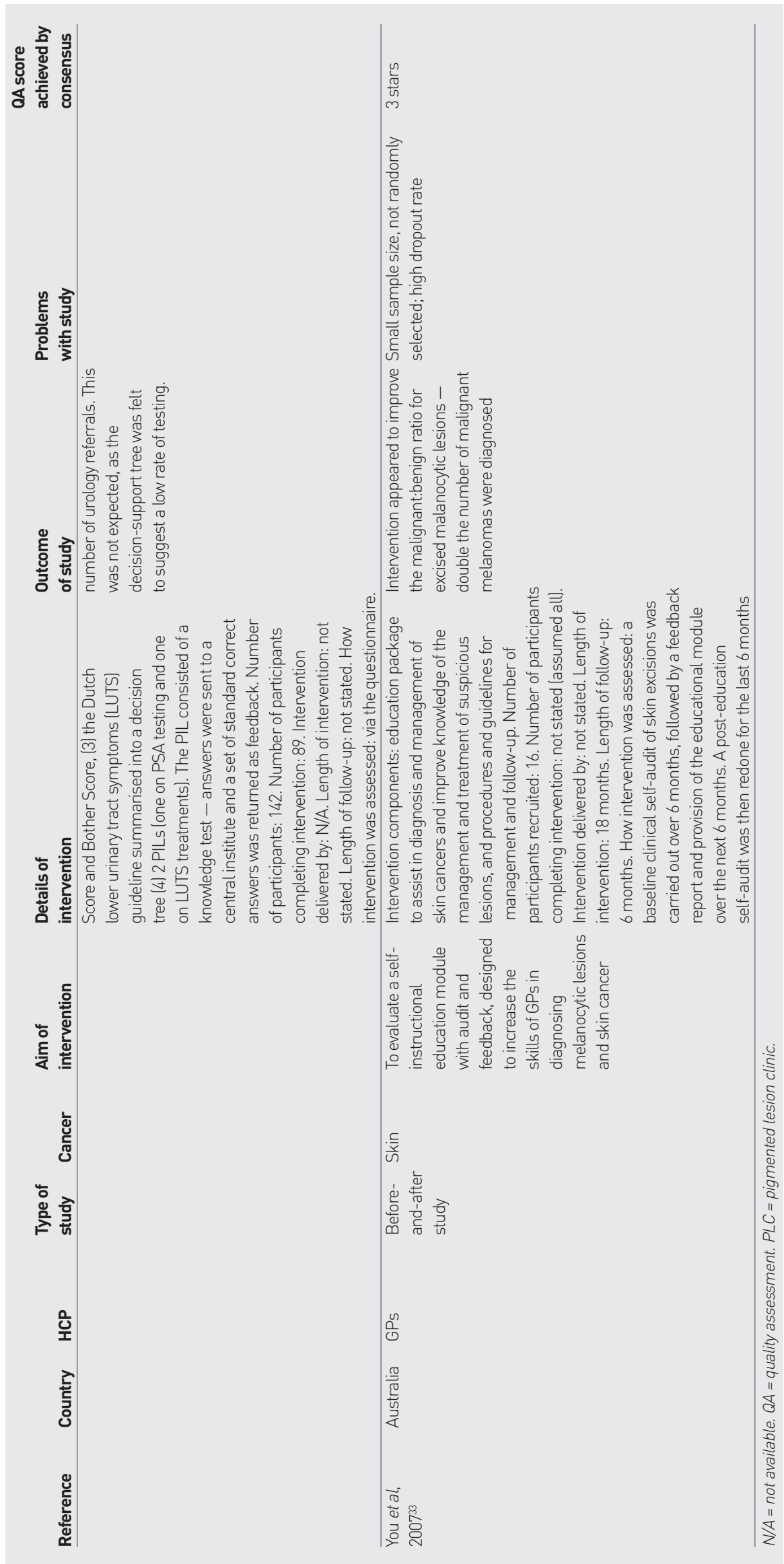

surface microscopy. ${ }^{31}$ The control group did not receive the training. Participants diagnostic ability on clinical photographs was found to significantly improve in those who had received the microscopy training.

Other specific skills training. Kirklin et al investigated whether arts-based skills could help improve observational skills. ${ }^{25}$ This lowquality RCT involved the intervention group being taught observational skills by an artist, while the control group received a traditional lecture on skin diseases. Observational skills were found to improve significantly more in the intervention group than the control group, which may lead to more accurate diagnoses in practice.

\section{DISCUSSION \\ Summary}

None of the studies identified had reduction in primary care delay in cancer referral as the primary outcome, and all used proxy measurements, many of which were not of clinical behaviour change. The evidence for the effectiveness of these studies was overall mostly positive, with 15 out of the 22 interventions having an impact on improving at least one of the outcomes measured. In contrast, the high-quality RCTs failed to show an improvement in aspects of management and knowledge using education, ${ }^{18,19}$ diagnostic accuracy using audit and feedback, ${ }^{28}$ and referral accuracy and investigation using decision-support software. 23,26 Mixed effects were found in aspects of management using educational material and a guideline. ${ }^{32}$ It was not always possible to pinpoint which particular aspects of the interventions led to particular improvements.

\section{Strengths and limitations}

Several problems were encountered in the studies included in this review. Some studies had a high risk of selection bias, $^{13-17,19,20,22,24,25,27,29-31}$ with little information given (if any) about whether those who participated were different from those who chose not to take part. Almost all studies reported a follow-up period of less than 1 year; $11,14,17-19,21,23,24,27,28,31,33$ several tested participants straight after the intervention; 15,16,29,30 and some did not include or state any follow-up period. 20,22,25,32 Therefore, it cannot be determined whether any of the reported improvements persisted. Less than half of the 22 studies included recruited more than 100 participants, $11,15,17,21,23,24,26,29,30,32$ and several studies suffered from a high dropout rate, $18,19,28,32,33$ with only $18 \%$ of participants in 
one study ${ }^{17}$ completing the intervention. These studies have low internal validity and may suggest that the intervention was not accepted by the PHPs. Among the RCTs, the randomisation process of some studies was either poorly reported or inadequate. ${ }^{13,18,21,22,25,26,28}$ In some cases, the control group may also have been sensitised to cancer through participation in the study, 18,19 in that their participation heightened their awareness of the topic. Generally, not enough detail was provided in the studies, especially with regard to information about the intervention and participants, to be able to assess the potential risk of bias.

Only papers published in English were included in this review, due to lack of resources for translation. Two papers the authors wished to examine in full could not be supplied by inter-library loan from the British Library. This led to four papers not being screened fully for inclusion. On closer examination of the article titles, abstracts, and journal titles of publication, it is unlikely these would have met the inclusion criteria and been included in the review.

\section{Comparison with existing literature}

In Austoker et al's review of studies to reduce patient delay, ${ }^{9}$ the authors concluded that interventions that are tailored to the particular group involved are more likely to improve outcomes. The present review provides some support for this, in that the types of intervention that appeared to be most effective were audit and feedback studies and specific skills training. Giving participants information specific to their practice appears to be more useful than giving general information on cancer-referral management. Other reviews and studies have focused on various methods to improve knowledge, such as continuing medical education ${ }^{10,13,35}$ and audit and feedback, ${ }^{36}$ which have given mixed evidence as to the effectiveness of these types of interventions, although in general there appears to be a small positive effect in improving professional practice and healthcare outcomes for patients. Mansouri and Lockyer concluded that more-effective interventions tend to be more interactive, use a range of methods, and be used with small groups. ${ }^{35}$

\section{Implications for research and practice}

While evidence is limited, audit and feedback and specific skills training appear to be the most effective in improving outcomes other than short-term ones, and the success of these studies and comparison with other existing literature indicate that interventions are most useful when tailored to the needs of the population being studied. No studies were found that explicitly measured delay as an outcome. This could be because of the considerable difficulties in undertaking cancer research in primary care due to the rarity of the disease in this population. Future research that attempts to measure delay directly is needed to determine which type of intervention is most effective in reducing the time for referral from primary to secondary care. Such studies require large participant populations and associated funding. This, in combination with improved reporting of methods and results, longer follow-up periods, and attempts to increase retention of participants, will be necessary to increase confidence in the conclusions of these studies and subsequent improvement in health care.

\section{Funding}

NHS North Staffordshire; NHS Stoke; NHS Executive West Midlands R\&D Office; North Staffordshire and Cheshire R\&D Consortium

\section{Provenance}

Freely submitted; externally peer reviewed.

\section{Competing interests}

The authors have declared no competing interests.

\section{Acknowledgements}

We wish to thank Drs Giri Rajaratnam, Judith Bell, Zafar lqbal, Max Kalsi, and Jackie Small, Professor Peter Croft, and Brian Dudley for commenting on the study design and draft paper.

\section{Discuss this article}

Contribute and read comments about this article on the Discussion Forum: http://www.rcgp.org.uk/bjgp-discuss 


\section{REFERENCES}

1. Richards MA, Westcombe AM, Love SB, et al. Influence of delay on survival in patients with breast cancer: a systematic review. Lancet 1999; 353(9159): 1119-1127.

2. Neal RD. Do diagnostic delays in cancer matter? Br J Cancer 2009; 101(suppl 2): S9-S12.

3. Neal RD, Allgar VL, Ali N, et al. Stage, survival and delays in lung, colorectal, prostate and ovarian cancer: comparision between diagnostic routes. $\mathrm{Br} \mathrm{J}$ Gen Pract 2007; 57(536): 212-219.

4. Macdonald S, Macleod U, Mitchell E, et al. Factors influencing patient and primary care delay in the diagnosis of cancer: a database of existing research and its implications for future practice. Report to the Department of Health, Cancer Symptom Profiles and Referral Strategies for Primary Care Research Programme 2004. Project Ref 1217522.

5. Department of Health. The NHS cancer plan. London: Department of Health, 2000

6. Richards M. EUROCARE-4 studies bring new data on cancer survival. Lancet 2007; 8(9): 752-753.

7. Souhami R. Are UK cancer cure rates worse than in most other European countries? Br J Gen Pract 2010; 60(571): 81-82.

8. Lewis R, Collins R, Flynn A, et al. A systematic review of cancer waiting time audits. Qual Saf Health Care 2005; 14(1): 62-66.

9. Austoker J, Bankhead C. Forbes LJ, et al. Interventions to promote cance awareness and early presentation: systematic review. Br J Cancer 2009; 101(Suppl 2): S31-S39.

10. Forsetlund $L$, Bjorndal $A$, Rashidian $A$, et al. Continuing education meetings and workshops: Effects of professional practice and health care outcomes (review). Cochrane Database Syst Rev 2009; (2): CD003030.

11. Nekhlyudov L, Nicola M, Jung I, Buechler E. Clinicians' knowledge and attitudes about breast symptom management: Is there a use for clinical guidelines? J Womens Health 2008; 17(1): 57-65.

12. Wells GA, Shea B, O'Connell D, et al. The Newcastle-Ottawa Scale (NOS) for assessing the quality of nonrandomised studies in meta-analyses. http://www.ohri.ca/programs/clinical_epidemiology/oxford.asp laccessed 29 Jun 2011).

13. Argenziano G, Puig S, Zalaudek I, et al. Dermoscopy improves accuracy of primary care physicians to triage lesions suggestive of skin cancer. J Clin Oncol 2006; 24(12): 1877-1882.

14. Bedlow AJ, Cliff S, Melia J, et al. Impact of skin cancer education on general practitioners' diagnostic skills. Clin Exp Dermatol 2000; 25(2): 115-118.

15. Brochez L, Verhaeghe E, Bleyen L, Naeyaert J-M. Diagnostic ability of general practitioners and dermatologists in discriminating pigmented skin lesions. $J$ Am Acad Dermatol 2001; 44(6): 979-986.

16. Carli $P, D e G$ V, Crocetti $E$, et al. Diagnostic and referral accuracy of family doctors in melanoma screening: Effect of a short formal training. Eur $J$ Cancer Prev 2005; 14(1): 51-55

17. Cockburn J, Pit S, Zorbas H, Redman S. Investigating breast symptoms in primary care: enhancing concordance with current best advice. Cancer Detect Prev 2001; 25(5): 407-413.

18. De Gannes GC, Ip JL, Martinka M, et al. Early detection of skin cancer by family physicians: a pilot project. J Cutan Med Surg 2004; 8(2): 103-109.

19. Dolan NC, Ng JS, Martin GJ, et al. Effectiveness of a skin cancer control educational intervention for internal medicine housestaff and attending physicians. J Gen Intern Med 1997; 12(9): 531-536.

20. Gerbert B, Bronstone A, Maurer T, et al. Decision support software to help primary care physicians triage skin cancer: a pilot study. Arch Dermatol 2000; 136(2): 187-192.

21. Gerbert B, Bronstone A, Maurer T, et al. The effectiveness of an internetbased tutorial in improving primary care physicians' skin cancer triage skills. J Cancer Educ 2002; 17(1): 7-11.

22. Girgis A, Sanson-Fisher RW, Howe C, Raffan B. A skin cancer training programme: Evaluation of a postgraduate training for family doctors. Med Educ 1995; 29(5): 364-371.

23. Jiwa M, Skinner $\mathrm{P}$, Coker $\mathrm{AO}$, et al. Implementing referral guidelines: lessons from a negative outcome cluster randomised factorial trial in general practice. BMC Fam Pract 2006; 7: 65

24. Khan NF. Implementation of a diagnostic tool for symptomatic colorectal cancer in primary care: a feasibility study. Prim Health Care Res Dev 2009; 10 $54-64$.

25. Kirklin D, Duncan J, McBride S, et al. A cluster design controlled trial of artsbased observational skills training in primary care. Med Educ 2007; 41(4): 395-402.

26. Logan ECM, Yates JM, Stewart RM, et al. Investigation and management of iron deficiency anaemia in general practice: a cluster randomised controlled trial of a simple management prompt. Postgrad Med J 2002; 78(923): 533537.

27. Moreno-Ramirez D, Ferrandiz L, Galdeano R, Camacho FM Teledermatoscopy as a triage system for pigmented lesions: a pilot study. Clin Exp Dermatol 2005; 31(1): 13-18

28. Raasch BA, Hays R, Buettner PG. An educational intervention to improve diagnosis and management of suspicious skin lesions. J Contin Educ Health Prof 2000; 20(1): 39-51.

29. Shah NM, Soliman AS, Banerjee M, et al. Knowledge gained after a brief CME module on breast cancer diagnosis. J Cancer Educ 2006; 21(3): 169-174.

30. Soliman AS, Samadi S, Banerjee M, et al. Brief Continuing Medical Education (CME) module raises knowledge of developing country physicians. International Electronic Journal of Health Education 2006; 9: 31-42.

31. Westerhoff K, McCarthy WH, Menzie SW. Increase in the sensitivity for melanoma diagnosis by primary care physicians using skin surface microscopy. Br J Dermatol 2000; 143(5): 1016-1020.

32. Wolters R, Wensing M, van Weel C, Grol R. The effect of a distance-learning programme on patient self-management of lower urinary tract symptoms (LUTS) in general practice: a randomised controlled trial. Eur Urol 2004. 46(1): 95-101.

33. Youl PH, Raasch BA, Janda M, Aitken JF. The effect of an educational programme to improve the skills of general practitioners in diagnosing melanocytic/pigmented lesions. Clin Exp Dermatol 2007; 32(4): 365-370.

34. Friedman RJ, Rigel DS, Kopf AW. Early detection of malignant melanoma: the role of physician examination and self-examination of the skin. CA Cancer J Clin 1985; 35(3): 130-151.

35. Mansouri M, Lockyer J. A meta-analysis of continuing medical education effectiveness. J Contin Educ Health Prof 2007: 27(1): 6-15.

36. Jamvedt G, Young JM, Kristoffersen DT, et al. Audit and feedback: effects on professional practice and health care outcomes (review). Cochrane Database Syst Rev 2006; (2): CD000259 